\title{
Andreas Engel \\ Microscopic assessment of membrane protein structure and function
}

Accepted: 3 July 2003 / Published online: 24 July 2003

(C) Springer-Verlag 2003

\begin{abstract}
Membrane proteins represent an important class of proteins that are encoded by about $40 \%$ of all genes, but compared to soluble proteins structural information is sparse. Most of the atomic coordinates currently available are from bacterial membrane proteins and have been obtained by X-ray crystallography. Recent results demonstrate the imaging power of the atomic force microscope and the accuracy of electron crystallography. These methods allow membrane proteins to be studied while embedded in the bilayer, and thus in a functional state. The low signal-to-noise ratio of cryoelectron microscopy is overcome by crystallizing membrane proteins in a two-dimensional protein-lipid membrane, allowing its atomic structure to be determined. In contrast, the high signal-to-noise ratio of atomic force microscopy allows individual protein surfaces to be imaged at subnanometer resolution, and their conformational states to be sampled. This review discusses examples of microscopic membrane protein structure determination and illuminates recent progress.
\end{abstract}

Keywords 3D electron microscopy - Atomic force microscopy $\cdot$ Electron diffraction - Membrane protein crystallization

\section{Introduction}

Biological membranes fulfill many vital functions as interfaces to the outside world, as interfaces between cells, and as boundaries of intracellular compartments.

Robert Feulgen Lecture 2003 presented at the 45th Symposium of the Society for Histochemistry in Les Diablerets, Switzerland, on 17 September 2003

\footnotetext{
A. Engel $(\varangle)$

M.E. Müller Institute for Microscopy, Biozentrum, University of Basel,

Klingelbergstrasse 70, 4056 Basel, Switzerland

e-mail: andreas.engel@unibas.ch

Tel.: +41-61-2672262

Fax: +41-61-2672109
}

Thus, biological membranes are related to numerous diseases such as hyperinsulinemia, nephrogenic diabetes insipidus, congestive heart failure, liver cirrhosis, cystic fibrosis, hyper- and hypotension, lung edema, epilepsy, retinitis pigmentosa, and cataract. About $40 \%$ of the sequenced genes appear to code for membrane proteins. However, only 40 membrane protein structures are available at atomic resolution, and among them only a few are mammalian proteins, compared to 4,000 unique crystal structures of soluble proteins. Therefore, progress in membrane structure determination is essential to enhance our understanding of biological membranes and their functions, and a prerequisite for rational drug design.

To assess the structure of soluble proteins at atomic resolution X-ray crystallography and NMR spectroscopy are the most efficient methods. In contrast, progress in structure determination of membrane proteins is slow, because of serious difficulties in producing three-dimensional (3D) crystals of these proteins. In spite of this, the rate of new structures determined by X-ray crystallography has increased (Chang et al. 1998; Doyle et al. 1998; Fu et al. 2000; Hunte et al. 2000; Lancaster 2001; Locher et al. 2002; Luecke et al. 1999; Pebay-Peyroula et al. 1997). Suitable 3D crystals of the first animal channel protein, bovine aquaporin-1, have been produced, and the structure has been solved to $2.2 \AA$ resolution (Sui et al. 2001). The strength of X-ray crystallography is the wellestablished technology that allows structures to be solved to high resolution with enormous efficiency, but this route is still risky as a result of the crystallization bottleneck. Solution NMR is the other established method to determine atomic structures. It does not require 3D crystals and allows the dynamics of a protein to be assessed. Furthermore, progress toward the structure determination of membrane proteins (Fernandez et al. 2001) and large complexes has been reported (Fiaux et al. 2002). However, difficulties with the stability of solubilized membrane proteins can be a problem.

A powerful alternative to determine the structure of a membrane protein is its reconstitution into two-dimensional (2D) crystals in the presence of lipids (Stahlberg et 
al. 2001a; Werten et al. 2002). This approach restores the native environment of membrane proteins and thus their biological activity. Cryoelectron microscopy allows the assessment of the 3D membrane protein structure at atomic resolution. The atomic force microscope depicts biological membranes in aqueous solutions, and permits tracking of the movement of single loops (Scheuring et al. 2002a). In addition, this instrument allows the topology of membrane proteins and their interaction with ligands to be assessed (Clausen-Schaumann et al. 2000; Fisher et al. 2000)

This review introduces these microscopy techniques and summarizes recent results acquired in our laboratory.

\section{Microscopy techniques}

\section{Atomic force microscopy}

The only instrument that can be operated in liquid, and which provides subnanometer resolution images as well as a superb signal-to-noise ratio, is the atomic force microscope (AFM; Binnig et al. 1986). The AFM measures the surface topography by raster scanning the sample laterally below a sharp stylus that is attached to a flexible cantilever. A servo system controls the scanner, displacing the sample vertically to keep the cantilever deflection constant. The optical detector resolves deflections of $0.1 \mathrm{~nm}$ or better, which corresponds to a force difference of typically $10-50$ piconewton $(\mathrm{pN})$. Thus, an AFM can contour the topography of a biological sample in aqueous solutions at forces comparable to those of molecular interactions. By electrostatically balancing the forces between tip and sample, topographs are recorded without sample deformation (Müller et al. 1999b), yielding a lateral resolution sometimes better than $0.5 \mathrm{~nm}$, and a vertical resolution of typically $0.1 \mathrm{~nm}$ (Stahlberg et al. 2001a). In combination with highresolution imaging, the AFM offers the unique possibility to manipulate biologically active macromolecules under physiological conditions (Fotiadis et al. 2002a). Moreover, the possibility to image native membranes with the AFM is of particular interest, as no other instrument allows such biosurfaces to be visualized at a resolution better than $2 \mathrm{~nm}$ under physiological conditions.

The sensitivity of the cantilever deflection detector has made single molecule force measurements with the AFM possible (Clausen-Schaumann et al. 2000; Fisher et al. 2000). Force-distance curves are recorded by vertically displacing the tip toward the sample until a single molecule is attached by contact adhesion. Forces developing during subsequent tip retraction reflect the induced unfolding pathway of the protein. Acquisition of highresolution images before and after 'unzipping' a biomolecule allows the damage produced to be directly visualized (Müller et al. 1999a; Oesterhelt et al. 2000; Scheuring et al. 2002b).

\section{Electron microscopy (EM)}

Transmission electron microscopes equipped with a fieldemission gun transfer the atomic scale structural information with only a small loss from the sample to the image, which represents a projection of the $3 \mathrm{D}$ potential distribution of the object. Meaningful information, however, can only be extracted when the sample is structurally preserved in spite of the vacuum within the electron optical system. Suitable methods produce biomolecules embedded in a thin vitrified water layer (Dubochet et al. 1988), or embedded in a layer of partially dried and frozen sugar (Hirai et al. 1999). A combination of staining the sample with a heavy metal salt to enhance the contrast, and freezing the sample to preserve the highresolution structure is found in the cryonegative stain sample preparation method (Adrian et al. 1998). Since all these samples are sensitive to the electron beam, images must be acquired at low electron doses. High-resolution data of proteins are recorded at doses below 5 electrons/ $\AA^{2}$, with the sample kept at $104 \mathrm{~K}$ with liquid nitrogen (Conway et al. 1993), or at doses below 20 electrons $/ \AA^{2}$, when the sample is kept at $4.3 \mathrm{~K}$ using liquid helium temperature (Fujiyoshi 1998). Such recording doses produce inherently noisy images, whose information must be extracted by image averaging. To exploit the information of each elastically scattered electron to the fullest extent, the use of a highly coherent electron beam produced by a field emission gun (FEG) is mandatory (Fujiyoshi 1998; van Heel et al. 2000).

Since images (rather than diffraction patterns) are recorded, thousands of single particle projections can be acquired, classified, aligned, and averaged (Frank 2002; van Heel et al. 2000). In this way the complete information for a full 3D reconstruction of the potential map is collected without producing any crystals of the particles. Single particle reconstruction has mainly been used for large soluble complexes such as the ribosome (Frank 2002; van Heel et al. 2000), but also for some solubilized membrane proteins (Bibby et al. 2001; Grigorieff 1998). The achievable resolution of single particle structure determination is now getting better than $1 \mathrm{~nm}$.

Images of 2D crystals of membrane proteins, however, allow the image signal to be extracted by crystallographic methods. Using this approach, the atomic structure of bacteriorhodopsin (bR) was first determined (Henderson et al. 1990), and refined (Grigorieff et al. 1996; Kimura et al. 1997; Mitsuoka et al. 1999a) using prototype FEG microscopes while keeping the sample at a few kelvin. Two other atomic structures of membrane proteins were subsequently solved using similar instruments: the lightharvesting complex of plants (Kühlbrandt et al. 1994) and human aquaporin-1 (Murata et al. 2000). Recent progress in 2D crystallization has delivered crystals of several transporters [NhaA (Williams 2000), OxlT (Hirai et al. 2002), and SecYEG (Breyton et al. 2002)], of the bacterial rotor of ATPase (Stahlberg et al. 2001b; Vonck et al. 2002), and of a ClC-type chloride channel (Mindell 


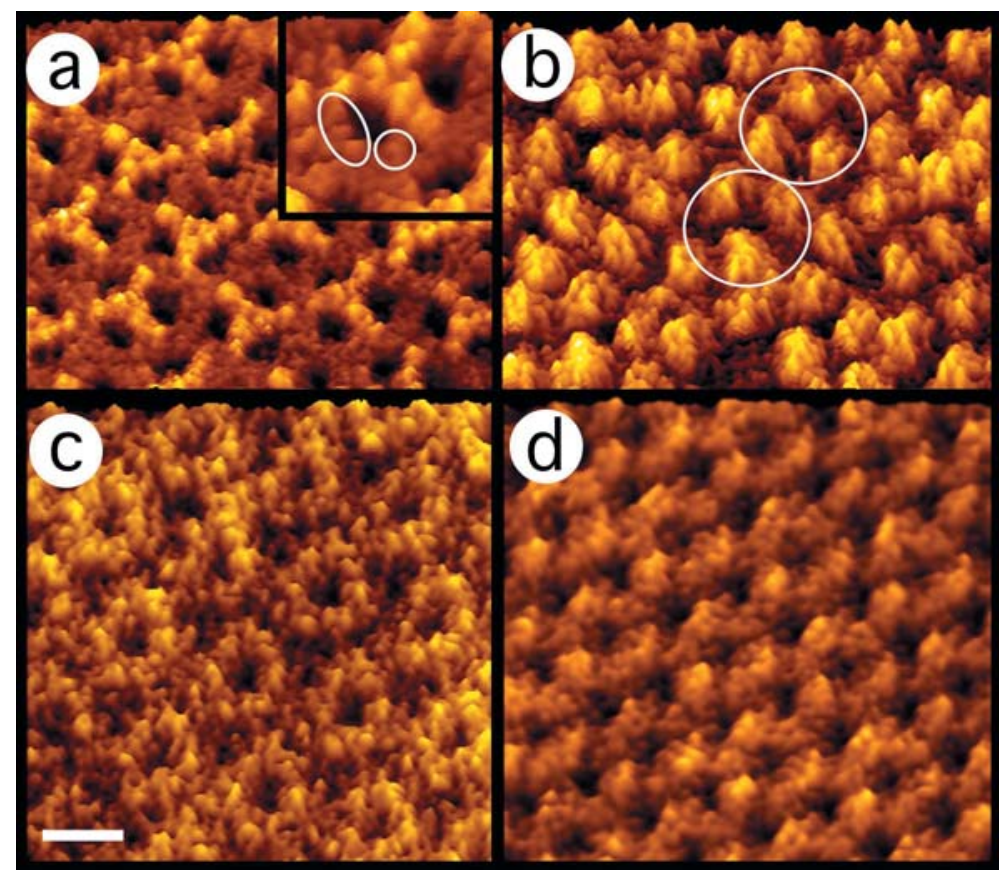

Fig. 1a-d Conformational changes of porin OmpF. a The features of the periplasmic OmpF surface, which are recognized in the unprocessed topograph recorded by atomic force microscopy in buffer solution, are fully interpretable based on the atomic structure (Cowan et al. 1992). Short $\beta$-turns comprising only a few amino acids are sometimes distinct (inset, circle, and ellipse). b Extracellular surface of OmpF. Two circles mark a trimer pair housed by one unit cell of the rectangular crystal. c pH-dependent conformational change of the extracellular surface. At $\mathrm{pH} \leq 3$ the flexible

et al. 2001), which allowed structure determination to a sufficient resolution (approximately $6 \AA$ ) to visualize the secondary structure.

\section{Bacterial porins}

Gram-negative bacteria exhibit a cell wall structure that includes an outer protecting membrane, a periplasmic space, and an inner membrane. Porins are proteinaceous pores that allow solutes to pass across the outer membrane. The structurally and functionally well-characterized porin $\mathrm{OmpF}$, a trimeric membrane channel, is a major outer membrane protein of $E$. coli. It comprises 16 antiparallel $\beta$ strands forming the transmembrane pore (Cowan et al. 1992). The strands are connected by short turns at the periplasmic (Fig. 1a and inset) and long loops at the extracellular surface, forming a domain that protrudes by $1.3 \mathrm{~nm}$ above the bilayer (Fig. 1b). An early puzzling finding is the voltage-gating properties of this channel: ions and solutes up to 600 Da pass unhindered up to an applied voltage of $150 \mathrm{mV}$ (Engel et al. 1985; Schindler and Rosenbusch 1978). While the reasons for weak ion selective properties of porins have been established based on its atomic structure (Schirmer and Phale 1999), the voltage-gating mechanism remained elusive until a series of AFM experiments provided loops reversibly collapse toward the center of the trimer thereby reducing their height from 1.3 to $0.6 \mathrm{~nm}$. d Conformational change of porin induced by an electrolyte gradient. The monovalent electrolyte gradient across the membrane was $>300 \mathrm{mM}$. Similar to the $\mathrm{pH}$-dependent conformational change, the extracellular domains reversibly collapsed onto the porin surface. Topographs exhibit a vertical range of $1(\mathbf{a}), 1.5(\mathbf{b})$, and $1.2 \mathrm{~nm}(\mathbf{c}, \mathbf{d})$ and the scale bar represents $5 \mathrm{~nm}$

further structural information. Three conditions were found to induce a displacement of the extracellular domain toward the trimer center, resulting in a structure with a height of only $0.6 \mathrm{~nm}$ (Müller and Engel 1999): (1) application of an electric potential $>200 \mathrm{mV}$ across the membrane, (2) acidic $\mathrm{pH}(<3$; Fig. 1c), and (3) generation of a $\mathrm{K}^{+}$gradient $>0.3 \mathrm{M}$ (Fig. 1d). The second condition suggests a protective function: $E$. coli cells passing through the acidic milieu of a stomach may survive longer by closing the outer membrane pores. The first condition, however, is compatible with results from black lipid membrane experiments, which demonstrated that porin is a voltage-gated channel (Schindler and Rosenbusch 1978). Recent black lipid membrane experiments carried out with maltoporin demonstrated the $\mathrm{pH}$-induced gating of this maltose-specific channel (Andersen et al. 2002), corroborating the $\mathrm{pH}$-induced conformational change observed by AFM.

\section{S-layers}

Another type of protective structure found in bacteria and archae is the surface (S)-layer, which is usually assembled from a few proteins arranged in a regular array that covers the cell surface (Sleytr 1997). As interfaces to the environment S-layers mediate adhesion to biotic and 

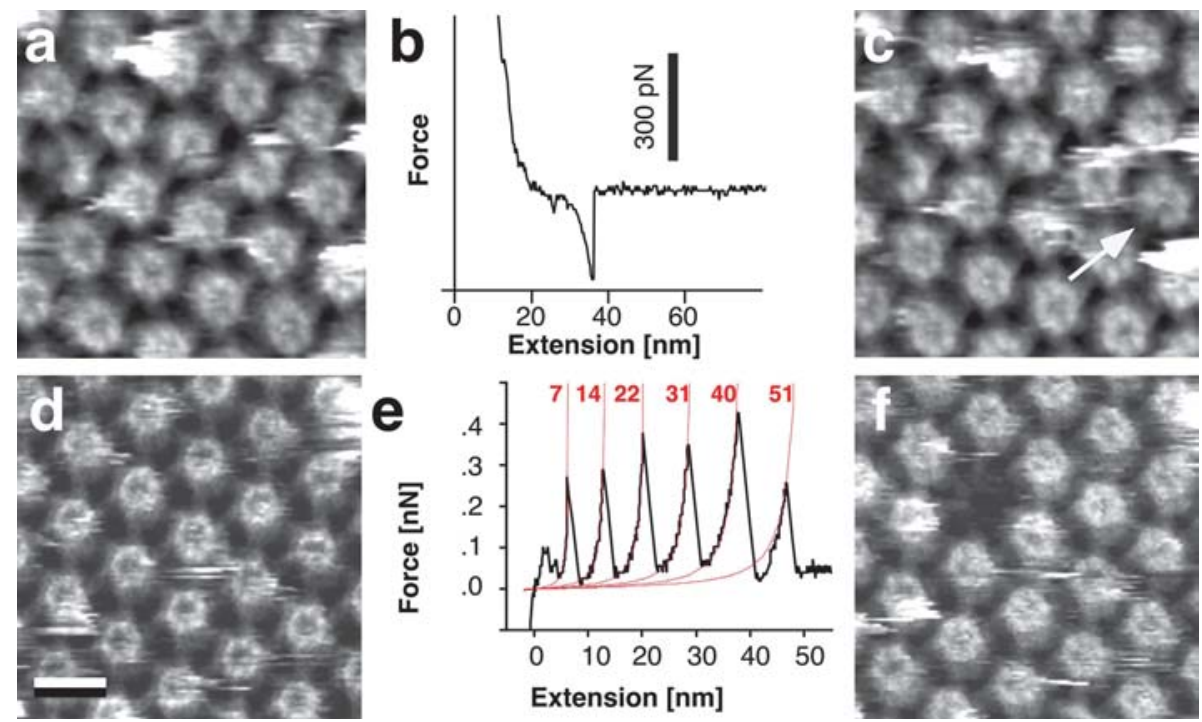

Fig. 2a-f Controlled unfolding of a hexagonally packed intermediate (HPI) hexamer. a The surface topography of the HPI-layer reveals hexameric cores that are connected by slender arms, which are connected at two-fold centers of symmetry. The central pore sometimes exhibits a central plug. b After formation of a molecular bridge between sample surface and tip, a force peak (approximately $300 \mathrm{pN}$ ) is sometimes observed upon retracting the stylus. c The topograph subsequently recorded reveals a striking change: one subunit is missing (arrow). d Another untouched area shows an

abiotic surfaces and allow uptake of nutrients. An extensively studied example is the hexagonally packed intermediate (HPI)-layer of Deinococcus radiodurans. Its structure has been assessed by both electron microscopy (Baumeister et al. 1986) and atomic force microscopy (Müller et al. 1996). Six identical protomers form the HPI-layer pore, which can reversibly switch from an unplugged to a plugged conformation (Müller et al. 1996). The outer surface of the HPI-layer is hydrophilic, whereas the hydrophobic N-terminal region of the HPI polypeptide (Peters et al. 1987), located at its inner surface, anchors the HPI-layer in the outer membrane. As a protective barrier, the HPI-layer exhibits an unusual resistance to extreme ionic strengths over a wide $\mathrm{pH}$ range, to aggressive detergents as well as to mechanical and to osmotic stress.

High-resolution topographs of the inner HPI-layer surface reveal cores with a central channel that are connected by slender arms (Fig. 2a). When approaching clean areas with the stylus until a force of $1 \mathrm{nN}$ is reached, a molecular bridge between stylus and HPI-layer is formed in 10-20\% of the approaches. Upon retraction of the stylus, characteristic force-extension curves exhibiting two types of force peaks are observed (Müller et al. 1999a). Peaks of the first type were typically $90 \mathrm{pN}$ high, and subsequent imaging of the same area did not reveal any structural change, indicating rupture of the molecular bridge between AFM stylus and HPI-layer. The second type had three times higher force peaks (Fig. 2b), and the topograph subsequently recorded showed a striking intact HPI-layer. e A rare event is the occurrence of multiple peaks, in this case six of them, rather similar in height and equally spaced [the lengths of the fitted worm-like chains (Fisher et al. 2000) are given in nanometers]. $\mathbf{f}$ The six distinct force peaks are related to the unzipping of an entire HPI hexamer, as documented by the topograph subsequently recorded. The molecular contaminants represent fiducial marks to identify the area scanned prior to deposition of the tip. Scale bar represents $20 \mathrm{~nm}$ and the brightness range corresponds to $7 \mathrm{~nm}$

change: a single protomer was always missing from one HPI hexamer, as illustrated by comparing the frame recorded before (Fig. 2a) and after the occurrence of a type II force peak (Fig. 2c arrow). A much less frequent event has a force-extension curve, which exhibits six major (200-300 pN) equally spaced peaks (Fig. 2e), while the corresponding control topograph shows that during retraction of the stylus an entire HPI hexamer has been zipped out (Fig. 2f; compare to Fig. 2d). Rupture events with two, three, four, or five peaks in the force-extension curve are also observed, and are correlated with the loss of two, three, four, or five monomers, respectively.

Equally spaced force peaks (Fig. 2e) indicate a strong interaction between protomers through a flexible link that has a length of $7.3 \pm 1.6 \mathrm{~nm}$, close to the thickness of the HPI-layer. The fact that several protomers can be pulled out sequentially implies that the interaction forces within hexamers are stronger than the forces between them. Thus, extraction of each protomer involves breakage of the spoke that connects hexamers within the HPI-layer (Müller et al. 1999a).

The S-layer of Corynebacterium glutamicum, another well-characterized S-layer, is assembled from the PS2 protein. High-resolution AFM topographs (Fig. 3a-d) of native and digested $\mathrm{S}$-layers reveal a hydrophilic, protease-resistant outer surface, and a hydrophobic, protease cleavable inner surface (Scheuring et al. 2002b). In spite of its hexameric nature, this S-layer exhibited a distinctly different unfolding pattern compared to the HPI-layer. Unzipping of a total hexameric core from C. glutamicum 


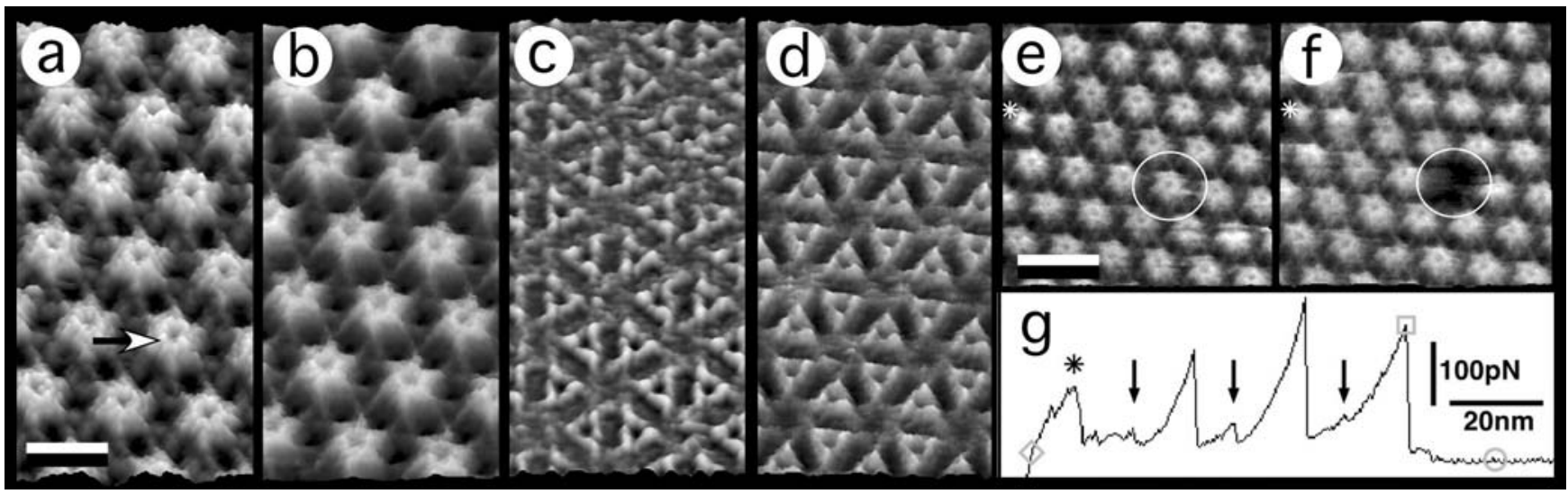

Fig. 3a-g Surface topography, proteolytic digestion, and unfolding of the S-layer of Corynebacterium glutamicum. a The inner surface reveals six-fold cores, which have a central pore and are connected by slender arms at three-fold centers of symmetry. A small crown (arrow) is distinct. b After trypsin cleavage, the crown is missing. c The extracellular surface shows triangular units arranged around the six-fold center of symmetry. d This extracellular surface is not affected by trypsin. e An almost perfect region of the S-layer

reveals three major rupture peaks (approximately $270 \mathrm{pN}$; Fig. 3e-g) interspersed by three faint peaks (approximately $70 \mathrm{pN}$; Scheuring et al. 2002b), in contrast to the six rupture peaks (approximately $300 \mathrm{pN}$ ) measured for the removal of one hexameric core of $D$. radiodurans. This difference of the force curves reflects the different packing arrangements of the two hexagonal protein lattices. While D. radiodurans HPI-layer proteins assemble into hexameric cores with each protein making one additional connection with one protomer of another core (Fig. 2), the S-layer proteins form hexameric cores with each protein being connected to two protomers of two other cores (Fig. 3). The six almost equal force peaks observed upon unzipping the HPI-layer are explained by strong interaction forces between subunits within the core and weaker forces (i.e., $300 \mathrm{pN}$ ) between the slender arms interacting at two-fold centers. In the $C$. glutamicum Slayer, however, subunits of adjacent cores are connected at three-fold centers.

\section{Rhodopsin}

Rhodopsin is the primary molecule in the visual signaling cascade. Activated by a single photon it induces subunit dissociation of the heterotrimeric transducin molecules, the cognate G-protein, which amplifies the light signal. Rhodopsin, whose structure has recently been solved, is thus a prototype G-protein-coupled receptor (GPCR) and a member of subfamily A comprising approximately $90 \%$ of all GPCRs. In the vertebrate retinal photoreceptors, rod outer segment-disk membranes are tightly stacked to ensure a dense packing of light absorbing rhodopsins, and in turn, a high probability of single photon absorption. The organization of rhodopsin in the native membrane is important because recent studies indicate that the regu- exhibits a single partially damaged core (asterisk). f After unfolding of a single hexamer, the missing core is distinct (circles). g The force-distance curve recorded during tip retraction shows three major and three minor (arrows) peaks. This particular force curve is related to the subunit interaction at the three-fold center of symmetry. Scale bars represent $20 \mathrm{~nm}$ and the brightness range is $3.5 \mathrm{~nm}$ in $\mathbf{a}, \mathbf{b}, \mathbf{e}$, and $\mathbf{f}$, and $2 \mathrm{~nm}$ in $\mathbf{c}$ and $\mathbf{d}$

lation of GPCRs and their interaction with the G-protein heterotrimer depend on their oligomeric state. To unveil the native supramolecular arrangement of rhodopsin, rod outer segment-disk membranes were isolated from mouse retinas and adsorbed to mica. Their topography was measured in buffer solution using an infrared laser AFM. Single-layered disk membranes had a circular shape, diameters between 0.9 and $1.5 \mu \mathrm{m}$, a thickness between 7 and $8 \mathrm{~nm}$, and either a soft or a rather stiff, highly textured surface (Fotiadis et al. 2003; Liang et al. 2003). The topography of the latter could be acquired at high magnification (Fig. 4) revealing rows of rhodopsin pairs densely packed in paracrystalline arrays. The distinct, densely packed double rows clearly demonstrate the dimeric nature of the native rhodopsin protein, supporting previous biochemical and pharmacological analyses that proposed GPCR dimerization and higher oligomerization (Rios et al. 2001). In contrast to indirect evidence and evolutionary trace analysis the topographs acquired with the AFM presented here show the rhodopsin dimers directly.

\section{Aquaporins}

Membrane channels specific for water and small nonionic solutes have been predicted to regulate the osmotic pressure a long time ago, and their existence was demonstrated by expression of aquaporin-1 (AQP1) in Xenopus oocytes (Preston et al. 1992). Water channels exist in bacteria, plants, and animals, forming a family which comprises over 300 members that are divided into the aquaporins (AQPs) and glycerol facilitators (GLPs). Their core, formed by six full transmembrane and two half helices, is highly conserved, with essential differences between AQPs and GLPs. However, loops A, C, 
Fig. 4a-c Atomic force microscopy of a native disk membrane adsorbed on mica and imaged in buffer solution (20 mM TRIS-HCl, pH 7.8, $150 \mathrm{mM} \mathrm{KCl}, 25 \mathrm{mM} \mathrm{MgCl} 2$ ). Topographs were acquired in contact mode at minimal loading forces $(\leq 100 \mathrm{pN})$. a Topography and paracrystalline organization of rhodopsin in the native membrane. b The calculated power spectrum of a reflects the regular arrangement of the rhodopsin molecules. c At higher magnification rhodopsin dimers (broken ellipse) forming the paracrystals become evident. An arrowhead marks an occasional rhodopsin monomer. Scale bars represent $50 \mathrm{~nm}$ in $\mathbf{a},(5 \mathrm{~nm})^{-1}$ in $\mathbf{b}$, and $15 \mathrm{~nm}$ in c. The brightness range in $\mathbf{a}$ and $\mathbf{c}$ corresponds to $1.6 \mathrm{~nm}$

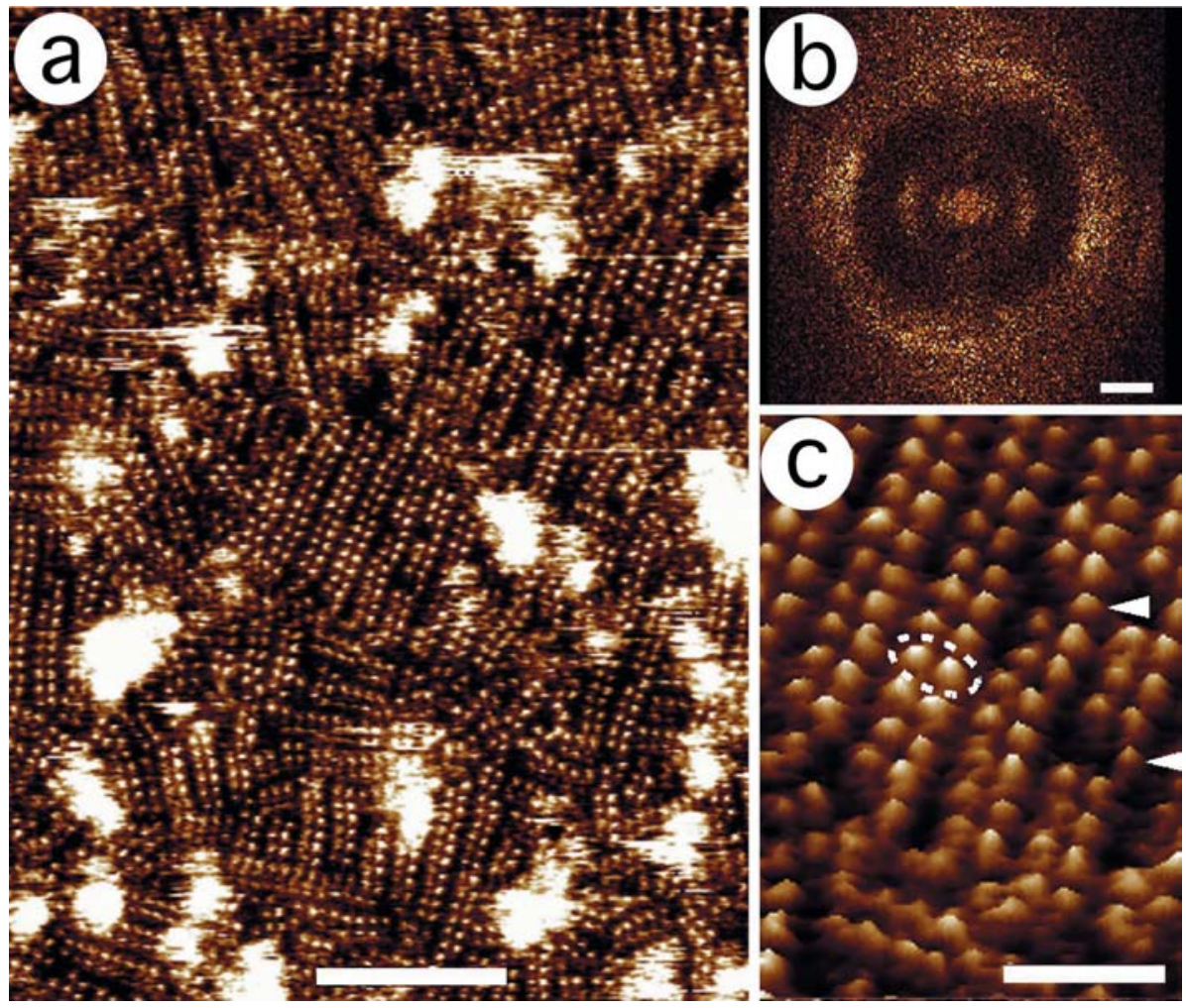

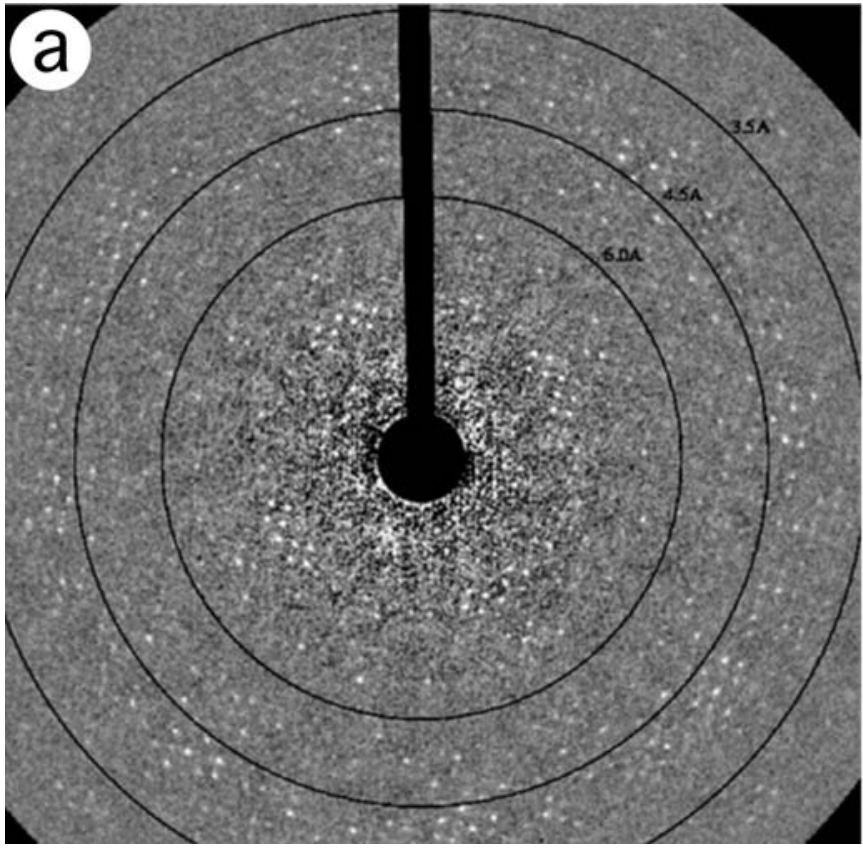

Fig. 5a, b Aquaporins readily crystallize when reconstituted in the bilayer at a lipid-to-protein ratio $<1$. The choice of the lipid, the $\mathrm{pH}$, and the presence of suitable counterions are critical. Electron

and $\mathrm{D}$, as well as the termini are variable, explaining different surface topographies, which have been revealed by AFM (Fotiadis et al. 2000, 2002b; Scheuring et al. 1999). Often, these structural features are related to function. For instance, adhesion of lens fiber cells is

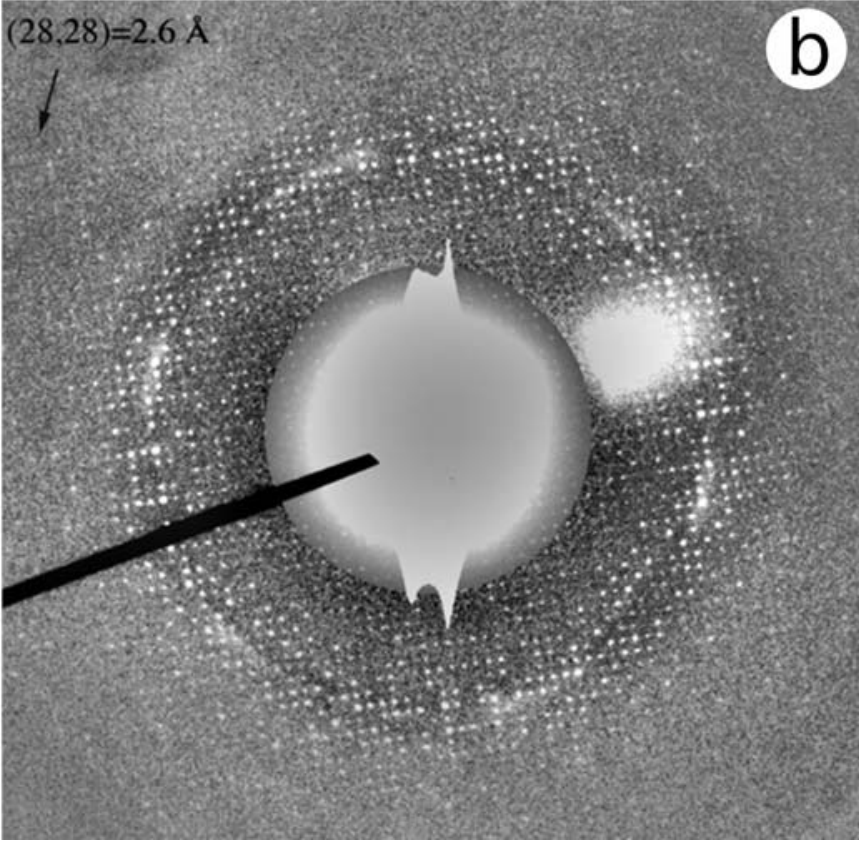

diffraction extending to $3 \AA$ resolution and beyond reveals high crystalline order for aquaporin (AQP)-1 (a; Mitsuoka et al. 1999b) and AQP2 (b)

likely to be mediated by AQP0, since knockout mice lacking AQP0 form irregular, highly scattering lens structures (Shiels et al. 2001). This is corroborated by AQP0 assembling into double-layered, precisely stacked 2D crystals resulting from a groove-and-tongue interac- 

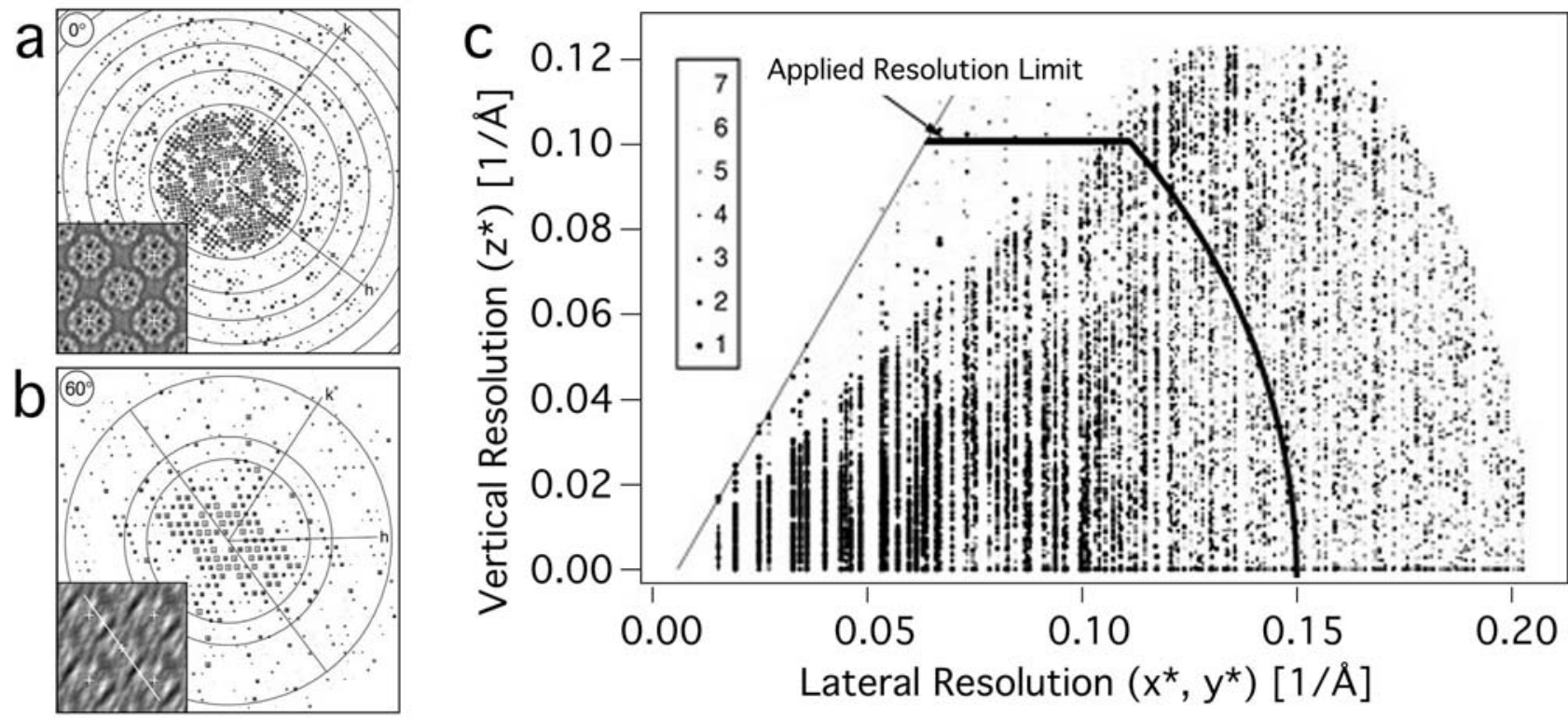

Fig. 6a-c Data collection and processing to build the $6.9 \AA$ potential map of GlpF (Stahlberg et al. 2000). a The projection map of GlpF tetramers recorded at a sample-tilt of $0^{\circ}$ together with the calculated Fourier transform. b The projection map of GlpF tetramers recorded at a sample-tilt of $60^{\circ}$ together with the

tion of protrusions at the extracellular surface (Fotiadis et al. 2000). Another example concerns the highly conserved C-terminus of AQP1, which harbors a putative $\mathrm{Ca}^{2+}$ binding site (EF-hand), whose function has yet to be elucidated (Fotiadis et al. 2002b). The AFM has also been used to sample the conformational states of loops connecting the helices of AqpZ, the E. coli water channel (Scheuring et al. 2002a).

While AFM depicts the surface features of densely packed membrane proteins under native conditions, their 3D structure can only be determined by electron or X-ray crystallography. Electron crystallography has provided the 3D structures of AQP1 (Murata et al. 2000) and of GlpF (Stahlberg et al. 2000). Electron crystallography offers the advantage to assess the structure of an aquaporin, whose function has been assessed in the crystallized state (Walz et al. 1994). A critical hurdle is the growth of highly ordered 2D crystals, which is in general more readily achieved than the growth of suitable $3 \mathrm{D}$ crystals. Aquaporins have a propensity to form 2D crystals as demonstrated by electron diffraction patterns of AQP1 and AQP2 (Fig. 5).

Once $2 \mathrm{D}$ crystals of high quality are available, electron crystallography allows the 3D potential map to be determined in a relatively straightforward manner (Fujiyoshi 1998). The procedure is illustrated in Fig. 6 for the GlpF structure determined to $6.9 \AA$ resolution (Stahlberg et al. 2000). First, projection maps are recorded from crystals that are tilted in the microscope between $0^{\circ}$ and $60^{\circ}$ (Fig. 6a, b). The respective Fourier coefficients are extracted from the calculated transforms and merged to calculated Fourier transform. c The merged data set used to establish the potential map at $6.9 \AA$ resolution. The data density is low between $45^{\circ}$ and $60^{\circ}$, but the information extends beyond the applied resolution limit

build up the 3D Fourier transform of the crystallized protein. The map displayed in Fig. 6c gives an account of the progress in data collection. When a resolution limit is applied as in Fig. 6c, the reconstructed potential map reveals essentially the topology of the protein; $\alpha$-helices can readily be accommodated (Fig. 7a). Upon reaching a higher resolution, as in the case of AQP1 (3.8 ̊; Murata et al. 2000), the structural detail suffices to build an atomic model (Fig. 7b). Further refinement yields a model (de Groot et al. 2001) that is sufficiently accurate to allow functional clues to be extracted (de Groot and Grubmüller 2001).

Atomic models derived from potential maps obtained by electron crystallography prior to X-ray crystallography provide an excellent opportunity to test the validity of EM-derived models. In the case of AQP1, instabilities during molecular dynamics simulation fostered a refinement of the atomic model (de Groot et al. 2001). The accuracy of this model was subsequently evaluated with the $2.2 \AA$ structure obtained by X-ray crystallography (Fig. 8), revealing an excellent correlation between the two structures (de Groot et al. 2003).

\section{Perspectives}

Microscopy techniques open important possibilities to assess the structure, dynamics, and function of membrane proteins. The examples presented here intend to demonstrate that these techniques, although used and developed in relatively few laboratories, now reach a level that 


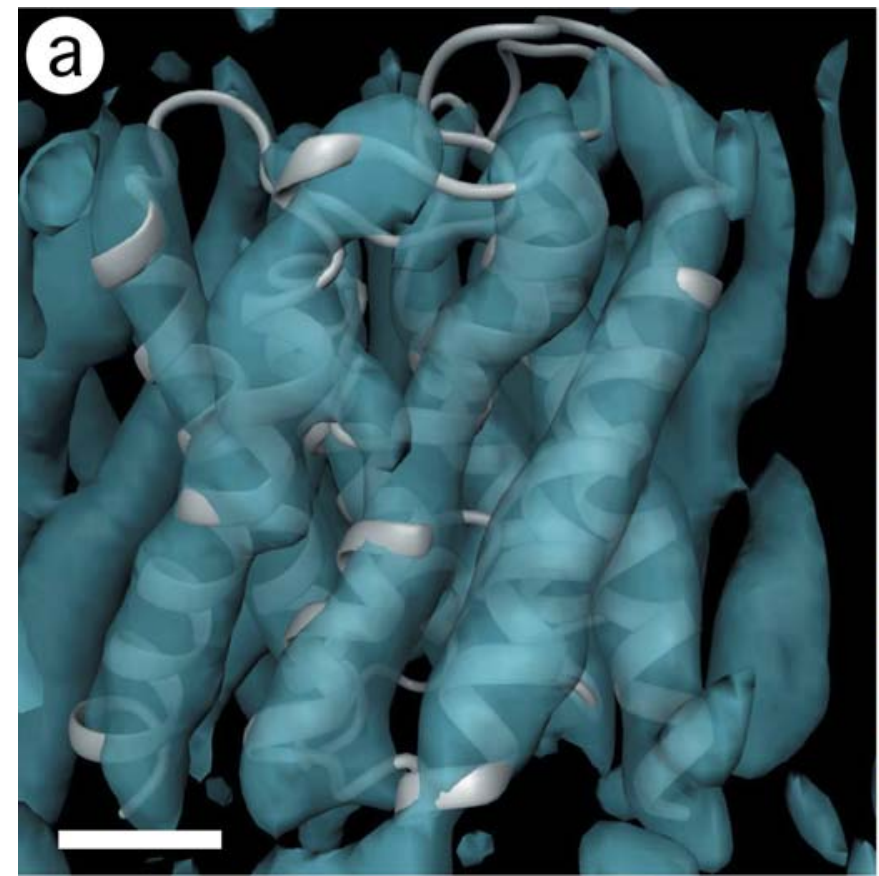

Fig. 7a, b Progress in the determination of the AQP1 atomic model. a At 6 A resolution the secondary structure becomes visible. b At $4.5 \AA$ resolution the peptide backbone can be placed, the side

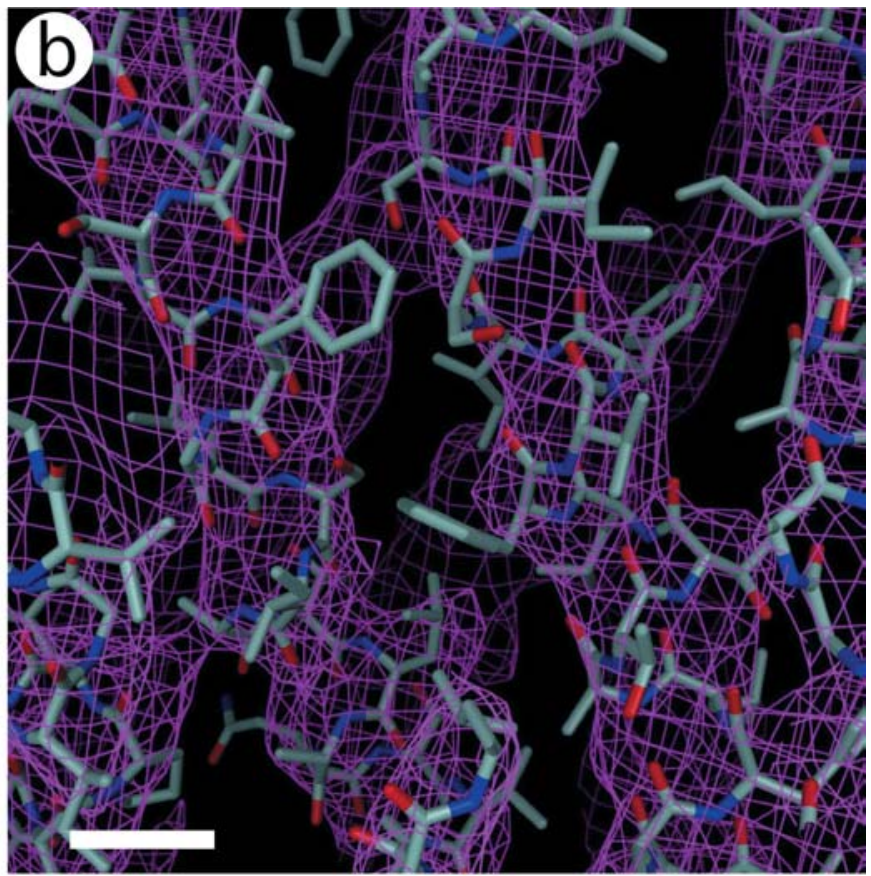

chains, however, are not discernible in the map. Scale bars represent $1 \mathrm{~nm}$ in $\mathbf{a}$ and $0.5 \mathrm{~nm}$ in $\mathbf{b}$

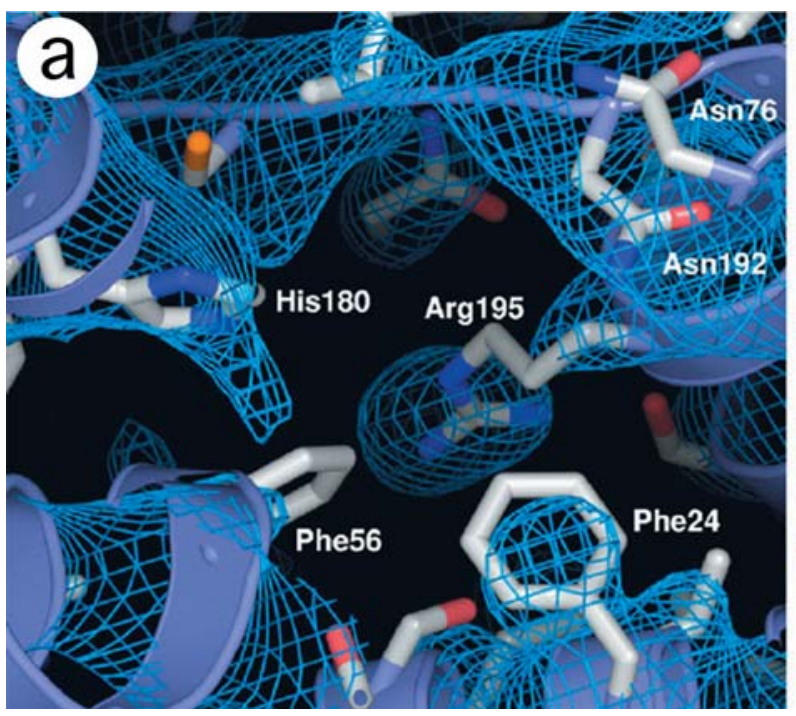

Fig. 8a, b Atomic models of AQP1. a The potential map of the channel region with the refined atomic model based on the $3.8 \mathrm{~A}$ resolution data obtained by electron crystallography. At this resolution, the definition of the side chains becomes clear, but the detailed packing arrangement needs to be refined by statistical

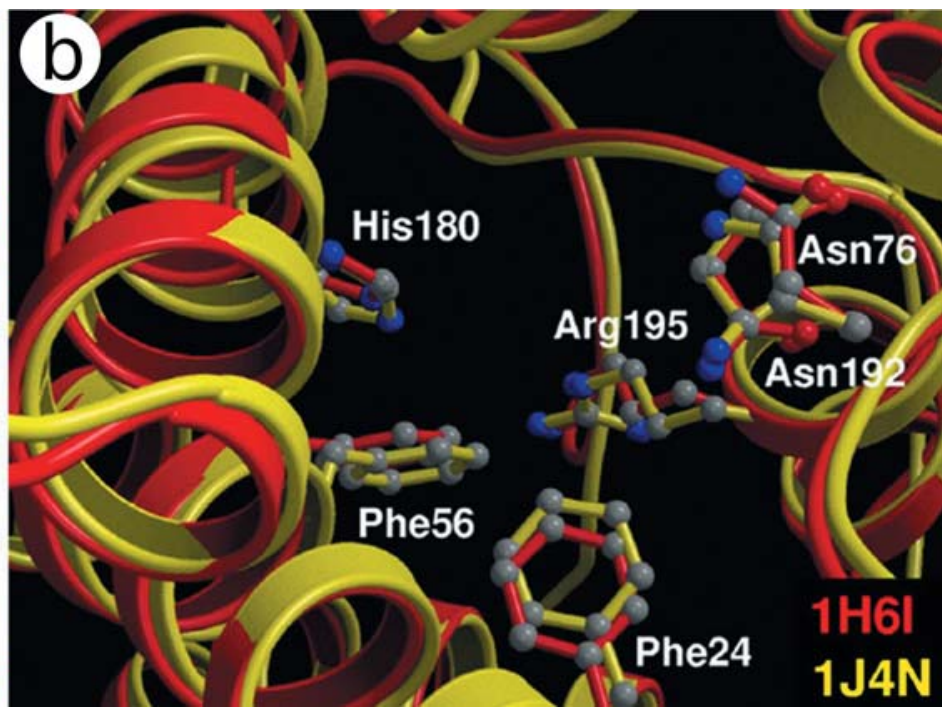

methods (de Groot et al. 2001). b Comparison of atomic models of AQP1 obtained by X-ray (yellow, bovine AQP1) and electron crystallography (red, human AQP1) reveals the close agreement of the independently determined structure (de Groot et al. 2003) allows their routine application. Compared to the efforts invested for X-ray crystallography in sample preparation (i.e., high-throughput 3D crystallization using robots) and the costs of synchrotrons as well as NMR instruments, the investment in microscopic techniques is rather small. Yet no other technique offers such a wide insight into the structure of living matter from the cellular up to the atomic level.

Here we have concentrated on discussing the highresolution end, where significant progress has been made. The results indicate that further developments of microscopy-based methods are important. The AFM will provide information about the native organization of biological 
membranes, the dynamics of membrane proteins, and their molecular interaction with ligands. Electron crystallography is expected to provide the atomic structure of membrane proteins that are not amenable to 3D crystallization. Ultimately, however, the same bottlenecks as found in X-ray crystallography will limit the progress of electron crystallography, namely, the large scale expression of functional membrane proteins and the problem of their crystallization.

Exciting progress has been reported in electron tomography, which now provides insight into the molecular organization of entire cells (Medalia et al. 2002). Combining the information gathered at all resolution levels will provide the structural knowledge required to understand not only the functioning of biomolecular complexes, but rather the working of complex biological systems.

Acknowledgements The research discussed here was supported by the Swiss National Research Foundation, the M.E. Müller Foundation, and the Swiss National Center of Competence in Research (NCCR) 'Structural Biology' and the NCCR 'Nanoscale Science.' The author is indebted to his creative and motivated collaborators and students, who have all contributed to the work summarized here. He also would like to thank Peter Agre, Yoshi Fujiyoshi, Helmut Grubmüller, and Kris Palczewski for most fruitful and inspiring collaborations and for their friendship.

\section{References}

Adrian M, Dubochet J, Fuller SD, Harris JR (1998) Cryo-negative staining. Micron 29:145-160

Andersen C, Schiffler B, Charbit A, Benz R (2002) pH-induced collapse of the extracellular loops closes Escherichia coli maltoporin and allows the study of asymmetric sugar binding. $\mathbf{J}$ Biol Chem 277:41318-41325

Baumeister W, Barth M, Hegerl R, Guckenberger R, Hahn M, Saxton WO (1986) Three-dimensional structure of the regular surface layer (HPI layer) of Deinococcus radiodurans. J Mol Biol 187:241-253

Bibby TS, Nield J, Barber J (2001) Three-dimensional model and characterization of the iron stress-induced CP43'-photosystem I supercomplex isolated from the cyanobacterium Synechocystis PCC 6803. J Biol Chem 276:43246-43252

Binnig G, Quate CF, Gerber C (1986) Atomic force microscope. Phys Rev Lett 56:930-933

Breyton C, Haase W, Rapoport TA, Kühlbrandt W, Collinson I (2002) Three-dimensional structure of the bacterial proteintranslocation complex SecYEG. Nature 418:662-665

Chang G, Spencer RH, Lee AT, Barclay MT, Rees DC (1998) Structure of the MscL homolog from Mycobacterium tuberculosis: a gated mechanosensitive ion channel. Science 282:22202226

Clausen-Schaumann H, Seitz M, Krautbauer R, Gaub HE (2000) Force spectroscopy with single bio-molecules. Curr Opin Chem Biol 4:524-530

Conway JF, Trus BL, Booy FP, Newcomb WW, Brown JC, Steven AC (1993) The effects of radiation damage on the structure of frozen hydrated HSV-1 capsids. J Struct Biol 111:222-233

Cowan SW, Schirmer T, Rummel G, Steiert M, Ghosh R, Pauptit RA, Jansonius JN, Rosenbusch JP (1992) Crystal structures explain functional properties of two E. coli porins. Nature 358:727-733

de Groot BL, Grubmüller H (2001) Water permeation across biological membranes: mechanism and dynamics of aquaporin1 and GlpF. Science 294:2353-2357 de Groot BL, Engel A, Grubmüller H (2001) A refined structure of human aquaporin-1. FEBS Lett 504:206-211

de Groot B, Engel A, Grubmüller H (2003) The structure of the aquaporin-1 water channel: a comparison between cryo-electron microscopy and X-ray crystallography. J Mol Biol 325:485-493

Doyle DA, Cabral JM, Pfuetzner RA, Kuo A, Gulbis JM, Cohen SL, Chait BT, MacKinnon R (1998) The structure of the potassium channel: molecular basis of $\mathrm{K}^{+}$conduction and selectivity. Science 280:69-77

Dubochet J, Adrian M, Chang J-J, Homo J-C, Lepault J, McDowall AW, Schultz P (1988) Cryo-electron microscopy of vitrified specimens. Q Rev Biophys 21:129-228

Engel A, Massalski A, Schindler H, Dorset DL, Rosenbusch JP (1985) Porin channel triplets merge into single outlets in Escherichia coli outer membranes. Nature 317:643-645

Fernandez C, Hilty C, Bonjour S, Adeishvili K, Pervushin K, Wuthrich K (2001) Solution NMR studies of the integral membrane proteins OmpX and OmpA from Escherichia coli. FEBS Lett 504:173-178

Fiaux J, Bertelsen EB, Horwich AL, Wüthrich K (2002) NMR analysis of a 900 K GroEL GroES complex. Nature 418:207211

Fisher TE, Marszalek PE, Fernandez JM (2000) Stretching single molecules into novel conformations using the atomic force microscope. Nat Struct Biol 7:719-724

Fotiadis D, Hasler L, Müller DJ, Stahlberg H, Kistler J, Engel A (2000) Surface tongue-and-groove contours on lens MIP facilitate cell-to-cell adherence. J Mol Biol 300:779-789

Fotiadis D, Scheuring S, Müller SA, Engel A, Müller DJ (2002a) Imaging and manipulation of biological structures with the AFM. Micron 33:385-397

Fotiadis D, Suda K, Tittmann P, Jeno P, Philippsen A, Müller DJ, Gross H, Engel A (2002b) Identification and structure of a putative $\mathrm{Ca}^{2+}$-binding domain at the $\mathrm{C}$ terminus of AQP1. J Mol Biol 318:1381-1394

Fotiadis D, Liang Y, Filipek S, Saperstein DA, Engel A, Palczewski K (2003) Atomic-force microscopy: rhodopsin dimers in native disc membranes. Nature 421:127-128

Frank J (2002) Single-particle imaging of macromolecules by cryoelectron microscopy. Annu Rev Biophys Biomol Struct 31:303-319

Fu D, Libson A, Miercke LJ, Weitzman C, Nollert P, Krucinski J, Stroud RM (2000) Structure of a glycerol-conducting channel and the basis for its selectivity. Science 290:481-486

Fujiyoshi Y (1998) The structural study of membrane proteins by electron crystallography. Adv Biophys 35:25-80

Grigorieff N (1998) Three-dimensional structure of bovine NADH:ubiquinone oxidoreductase (complex I) at $22 \AA$ in ice. J Mol Biol 277:1033-1046

Grigorieff N, Ceska TA, Downing KH, Baldwin JM, Henderson R (1996) Electron-crystallographic refinement of the structure of bacteriorhodopsin. J Mol Biol 259:393-421

Henderson R, Baldwin JM, Ceska TA, Zemlin F, Beckmann E, Downing KH (1990) Model for the structure of bacteriorhodopsin based on high-resolution electron cryo-microscopy. J Mol Biol 213:899-929

Hirai T, Murata K, Kimura Y, Fujiyoshi Y (1999) Trehalose embedding technique for high-resolution electron crystallography: application to structural study on bacteriorhodopsin. J Electron Microsc 48:653-685

Hirai T, Heymann JA, Shi D, Sarker R, Maloney PC, Subramaniam S (2002) Three-dimensional structure of a bacterial oxalate transporter. Nat Struct Biol 9:597-600

Hunte C, Koepke J, Lange C, Rossmanith T, Michel H (2000) Structure at $2.3 \AA$ resolution of the cytochrome bc(1) complex from the yeast Saccharomyces cerevisiae co-crystallized with an antibody $\mathrm{F}_{\mathrm{v}}$ fragment. Structure Fold Des 8:669-684

Kimura Y, Vassylyev DG, Miyazawa A, Kidera A, Matsushima M, Mitsuoka K, Murata K, Hirai T, Fujiyoshi Y (1997) Surface of bacteriorhodopsin revealed by high-resolution electron crystallography. Nature 389:206-211 
Kühlbrandt W, Wang DN, Fujiyoshi Y (1994) Atomic model of plant light-harvesting complex by electron crystallography. Nature 367:614-621

Lancaster CR (2001) Succinate:quinone oxidoreductases: what can we learn from Wolinella succinogenes quinol:fumarate reductase? FEBS Lett 504:133-141

Liang Y, Fotiadis D, Filipek S, Saperstein DA, Palczewski K, Engel A (2003) Organization of the $G$ protein-coupled receptors rhodopsin and opsin in native membranes. J Biol Chem 278:21655-21662

Locher KP, Lee AT, Rees DC (2002) The E. coli BtuCD structure: a framework for $\mathrm{ABC}$ transporter architecture and mechanism. Science 296:1091-1098

Luecke H, Schobert B, Richter HT, Cartailler JP, Lanyi JK (1999) Structure of bacteriorhodopsin at $1.55 \AA$ resolution. J Mol Biol 291:899-911

Medalia O, Weber I, Frangakis AS, Nicastro D, Gerisch G, Baumeister W (2002) Macromolecular architecture in eukaryotic cells visualized by cryoelectron tomography. Science 298:1209-1213

Mindell JA, Maduke M, Miller C, Grigorieff N (2001) Projection structure of a ClC-type chloride channel at $6.5 \AA$ resolution. Nature 409:219-223

Mitsuoka K, Hirai T, Murata K, Miyazawa A, Kidera A, Kimura Y, Fujiyoshi Y (1999a) The structure of bacteriorhodopsin at $3.0 \AA$ resolution based on electron crystallography: implication of the charge distribution. J Mol Biol 286:861-882

Mitsuoka K, Murata K, Walz T, Hirai T, Agre P, Heymann JB, Engel A, Fujiyoshi Y (1999b) Short-helices in hourglass poreforming domains of AQP1 water channel protein visualized at 4.5 Å. J Struct Biol 128:34-43

Müller DJ, Engel A (1999) Voltage and pH-induced channel closure of porin OmpF visualized by atomic force microscopy. J Mol Biol 285:1347-1351

Müller DJ, Baumeister W, Engel A (1996) Conformational change of the hexagonally packed intermediate layer of Deinococcus radiodurans imaged by atomic force microscopy. J Bacteriol 178:3025-3030

Müller DJ, Baumeister W, Engel A (1999a) Controlled unzipping of a bacterial surface layer with an AFM. Proc Natl Acad Sci U S A 96:13170-13174

Müller DJ, Fotiadis D, Scheuring S, Müller SA, Engel A (1999b) Electrostatically balanced subnanometer imaging of biological specimens by atomic force microscope. Biophys J 76:11011111

Murata K, Mitsuoka K, Hirai T, Walz T, Agre P, Heymann JB, Engel A, Fujiyoshi Y (2000) Structural determinants of water permeation through aquaporin-1. Nature 407:599-605

Oesterhelt F, Oesterhelt D, Pfeiffer M, Engel A, Gaub HE, Müller DJ (2000) Unfolding pathways of individual bacteriorhodopsins. Science 288:143-146

Pebay-Peyroula E, Rummel G, Rosenbusch JP, Landau EM (1997) X-ray structure of bacteriorhodopsin at 2.5 ångstroms from microcrystals grown in lipidic cubic phases. Science 277:16761681

Peters J, Peters M, Lottspeich F, Schäfer W, Baumeister W (1987) Nucleotide sequence of the gene encoding the Deinococcus radiodurans surface protein, derived amino acid sequence, and complementary protein chemical studies. J Bacteriol 169:52165223
Preston GM, Carroll TP, Guggino WB, Agre P (1992) Appearance of water channels in Xenopus oocytes expressing red cell CHIP28 protein. Science 256:385-387

Rios CD, Jordan BA, Gomes I, Devi LA (2001) G-protein-coupled receptor dimerization: modulation of receptor function. Pharmacol Ther 92:71-87

Scheuring S, Ringler P, Borgnia M, Stahlberg H, Müller DJ, Agre P, Engel A (1999) High resolution AFM topographs of the Escherichia coli water channel aquaporin Z. EMBO J 18:49814987

Scheuring S, Müller DJ, Stahlberg H, Engel HA, Engel A (2002a) Sampling the conformational space of membrane protein surfaces with the AFM. Eur Biophys J 31:172-178

Scheuring S, Stahlberg H, Chami M, Houssin C, Rigaud JL, Engel A (2002b) Charting and unzipping the surface layer of Corynebacterium glutamicum with the atomic force microscope. Mol Microbiol 44:675-684

Schindler H, Rosenbusch JP (1978) Matrix protein from Escherichia coli outer membranes forms voltage-controlled channels in lipid bilayers. Proc Natl Acad Sci U S A 75:3751-3755

Schirmer T, Phale PS (1999) Brownian dynamics simulation of ion flow through porin channels. J Mol Biol 294:1159-1167

Shiels A, Bassnett S, Varadaraj K, Mathias R, Al-Ghoul K, Kuszak J, Donoviel D, Lilleberg S, Friedrich G, Zambrowicz B (2001) Optical dysfunction of the crystalline lens in aquaporin-0 deficient mice. Physiol Genomics 7:179-186

Sleytr UB (1997) Basic and applied S-layer research: an overview. FEMS Microbiol Rev 20:5-12

Stahlberg H, Braun T, de Groot B, Philippsen A, Borgnia MJ, Agre P, Kuhlbrandt W, Engel A (2000) The 6.9-A structure of GlpF: a basis for homology modeling of the glycerol channel from Escherichia coli. J Struct Biol 132:133-141

Stahlberg H, Fotiadis D, Scheuring S, Remigy H, Braun T, Mitsuoka K, Fujiyoshi Y, Engel A (2001a) Two-dimensional crystals: a powerful approach to assess structure, function and dynamics of membrane proteins. FEBS Lett 504:166-172

Stahlberg H, Müller DJ, Suda K, Fotiadis D, Engel A, Meier T, Matthey U, Dimroth P (2001b) Bacterial Na(+)-ATP synthase has an undecameric rotor. EMBO Rep 2:229-233

Sui H, Han BG, Lee JK, Walian P, Jap BK (2001) Structural basis of water-specific transport through the AQP1 water channel. Nature 414:872-878

van Heel M, Gowen B, Matadeen R, Orlova EV, Finn R, Pape T, Cohen D, Stark H, Schmidt R, Schatz M, et al (2000) Singleparticle electron cryo-microscopy: towards atomic resolution. Q Rev Biophys 33:307-369

Vonck J, von Nidda TK, Meier T, Matthey U, Mills DJ, Kühlbrandt W, Dimroth P (2002) Molecular architecture of the undecameric rotor of a bacterial $\mathrm{Na}(+)$-ATP synthase. J Mol Biol 321:307-316

Walz T, Smith BL, Zeidel ML, Engel A, Agre P (1994) Biologically active 2-dimensional crystals of aquaporin CHIP. J Biol Chem 269:1583-1586

Werten PJ, Remigy HW, de Groot BL, Fotiadis D, Philippsen A, Stahlberg H, Grubmüller H, Engel A (2002) Progress in the analysis of membrane protein structure and function. FEBS Lett 529:65-72

Williams KA (2000) Three-dimensional structure of the ioncoupled transport protein NhaA. Nature 403:112-115 